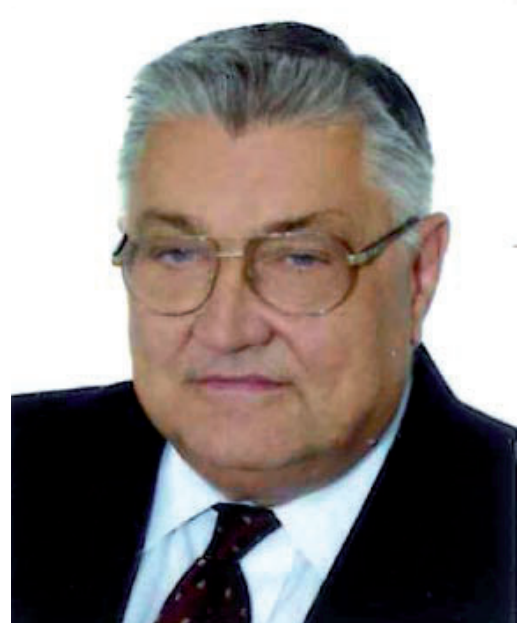

\title{
RECOLLECTION OF THE AVIATION PHYSIOLOGY LABORATORY AT MILITARY INSTITUTE OF AVIATION MEDICINE FROM THE PERSPECTIVE OF 50 YEARS OF PILOT PERFORMANCE RESEARCH
}

Bronisław TURSKI

Source of support: Own sources

Author's address: B.Turski, Warsaw, Poland, e-mail: bronislaw.turski@wiml.waw.pl

Abstract: The author after graduating from the Medical Military Academy in 1965, he was sented for a postgraduate two-year internship in medicine at the Military Institute of Aviation Medicine (WIML), which he partly completed there and partly at the Second Central Clinical Hospital of the Medical Military Academy (WAM). After the internship he was sented to the Air Force Institute of Technology to work in the position of a senior physician of the infirmary. After less than two years in 1969 he was transferred to the position of an assistant professor to the Laboratory of Aviation Physiology at MIAM. The paper deals with the characteristics of scientific and application activities of the Laboratory of Aviation Physiology at WIML in the years 1969-1996. The subject of research was, among others, the assessment of physical performance and its impact on the effectiveness of the operation of pilots and cosmonauts in extreme conditions. The research was carried out with the use of many flight simulators, such as: thermo- and diving chamber, centrifuge, and also in laboratory conditions (e.g. stationary bikes) and in water (lakes) or mountain (Tatra Mountains) natural conditions. Moreover, the effects of vibrations on the functioning of helicopter pilots were also dealt with. As part of space medicine, a prototype of a vacuum capsule was created, the essential element of which was a "vacuum collar" (mounted around a pelvis of the tested person), enabling the capsule to be under-pressurized to the required level at the lower half of the body.

Keywords: aeronautical and space physiology, body fitness, flight simulators 


\section{INTRODUCTION}

The paper deals with the characteristics of scientific and application activities of the Laboratory of Aviation Physiology at Military Institute Aviation Medicine (MIAM) in the years 1969-1996. The subject of research was, among others, the assessment of physical performance and its impact on the effectiveness of the operation of pilots and cosmonauts in extreme conditions. The research was carried out with the use of many flight simulators, such as: thermo- and diving chamber, centrifuge, and also in laboratory conditions (e.g. stationary bikes) and in water (lakes) or mountain (Tatry Mountains) natural conditions. Moreover, the effects of vibrations on the functioning of helicopter pilots were also dealt with. As part of space medicine, a prototype of a vacuum capsule was created, the essential element of which was a "vacuum collar" (mounted around a pelvis of the tested person), enabling the capsule to be underpressurized to the required level at the lower half of the body.

\section{A BRIEF OUTLINE OF THE HISTORICAL LABORATORY}

I trained and broadened my aviation and medical knowledge under the direction of Zbigniew Sarol, MD. At that time I was directed to the performance laboratory, where the methodology of physical performance testing on a stationary bike was introduced and where under the direction of Zbigniew Dziuk, MD. and in cooperation with Krzysztof Klukowski, MD. we conducted military research on aircraft personnel, using the methodology of physical performance assessment according to Astrand, Sjöstrand, the precursors of this research. The assessment of physical performance was based on a study of oxygen uptake during dosed physical activity calculated according to a formula that was modified by applying an age factor that took into account the Polish population. An assessment table for performance of aviation personnel was developed, which has been taken into account in the assessment of the general health status during the periodical examinations at the Aviation and Medical Commission. This type of research was carried out during the fitness camps, which were held alternately in Gronik/Zakopane and Mrągowo in Mazury.

In addition to performance and fitness tests, MIAM also provided training and preparatory courses for the aviation medicine specialization. These allowed me to obtain first and then second degree of specialization in aviation medi- cine. At the same time, the pilot's body tolerance to hypoxia was tested by performing various types of tests in the low-pressure chamber, using different methodologies. The basic method was a halfhour stay of the pilot in the chamber on a simulator of an altitude of $5000 \mathrm{~m}$ (pressure of $405 \mathrm{mmHs}$ ). Experimental tests of physical performance were also performed at various simulated altitudes in the low-pressure chamber with simultaneous exposure to low or high ambient temperatures. The result of this research was a doctoral thesis entitled "Effects of altitude hypoxia and low ambient temperatures on the pilot's body" (1975).

\section{RESEARCH APPARATUS IN THE LABORATORY AND RESEARCHES}

MIAM had at their disposal several chambers, including a diving chamber where 12 pilots could be tested simultaneously. Moreover, the chamber center had a thermo-diving chamber, which allowed to obtain high values of hypoxia and at the same time, depending on the needs, it could be combined with high or low temperatures even of $+/-47^{\circ} \mathrm{C}$. An important element of the aviation test was the barofunctional examination, i.e. obtaining the appropriate height in a short time and relatively quick descent from high altitudes to the normal ground pressure. The so-called rapid ascent and descent made it possible to assess the condition of the auditory system, especially after acoustic injuries or chronic inflammatory infections. Under the supervision of very experienced doctors in the field of chambers - assistant professor Eugeniusz Sokołowski, assistant professor Lucjan Golec, scientific experiments of various types were performed on animals and the research of aviation personnel could planned with precision. Research was developed on the so-called reserve time, i.e. on human tolerance to hypoxia. The research consisted in breathing a mixture with a small amount of oxygen, corresponding to an altitude of up to $7000 \mathrm{~m}$. The psychological test concerning visual attention consisted in subtraction of numbers from 1000. The errors that occurred indicated the amount of reserve time of the examined person until the moment of loss of consciousness. The results of these studies correlated with the results in the low pressure chamber.

An important element of aviation-medical research was the determination of overload tolerance of aviation personnel in various programmes - linear or intermittent. Depending on the needs, the programmes which were an element of a com- 
prehensive assessment of the psychological and physiological condition of the flying staff were applied. Both the results of tests in low pressure chambers and on overload centrifuges as well as tests of physical performance were assessed together with the results of clinical trials as a complex assessment of the health condition of each pilot.

At the same time, the routine test for flying personnel was to determine the overload tolerance. Adequate overload tolerance, especially in the $+\mathrm{Gz}$ axis (head - lower limbs), was an important element in assessing the orthostatic tolerance of a pilot and their suitability for the profession of a pilot. Among the scientific studies undertaken, an important topic in aviation in the 1970s were vibrations, occurring mainly on helicopters. They became a challenge, as some pilots often reported ailments, resembling the so-called vibration syndrome. Research of the flying personnel was undertaken, especially of helicopter pilots, where vibrations sometimes exceeded the standards in individual helicopter locations - floor, steering stick, pilot's seat. During the periodical examinations, plethysmographic examinations of the upper and lower limbs were performed, combined with the reaction of the vessels to low and high temperatures, in order to check the reactivity of the peripheral vessels under specific conditions. Moreover, a palesthesiometric test was used - the vibration sensing threshold test. Research was also carried out in aircraft facilities, e.g. in Pruszcz Gdański, where Mi-24 helicopters were stationed. The tests mentioned above were carried out prior to and shortly after the flights. The vibration intensity was measured at various points of the helicopter. Excessive vibration levels were found on the deck, on the control stick and on the pilot's seat. An intervention at the manufacturer (USSR) resulted in improvement of these conditions. The results of tests at MIAM as well as aircraft facility confirmed an increased reactivity of peripheral vessels, however, no symptoms typical for vibration syndrome were found. During this time, the average annual helicopter flight time did not exceed 150 hours, which resulted in the absence of clear symptoms of disease. In addition to the intensity of vibrations, the time of exposure to vibrations, which in this case was small and could not cause significant lesions, plays an important role in the occurrence of symptoms.

As the head of the vibration laboratory, I have developed a multidisciplinary methodology for assessing the effects of harmful vibrations. In this study, peripheral cardiovascular tests (plethys- mography, skin thermometry), palesthesiometry, EMG, eye fluorescein angiography, biochemical tests (free fatty acids, cholesterol), hormonal evaluation and bone radiograms were carried out. Conclusions from this study were used for an in-depth analysis of the mechanisms of changes in vascular, nervous, osteo-articular and hormonal systems. Among other things, proper work hygiene was applied, observing the hours of exposure to vibrations during flights, and after intensive flights, rehabilitation procedures were applied for pilots. A rehabilitation office for pilots was established in Pruszcz Gdański. In the 1970s, my main interests in aviation medicine were focused on the influence of overloads affecting various parts of the body during flights on new type of supersonic aircraft (Mig-23, Su-7).

\section{THE CHALLENGES OF GRAVITY MEDICINE}

Moreover, just before the flight into space of the Polish cosmonaut, I took part in the preparations of the entire group of candidates of ten members, who were subjected to complex, widely programmed research. This several months' long health selection carried out by dr. Krzysztof Klukowski, selected two candidates who successfully passed all the tests and verifications.

Mjr Mirosław Hermaszewski's space flight was the success of many doctors at MIAM. At the beginning of the 1980s, WIML engaged in a subsequent, broad scientific topic. As part of this problem, it was necessary to create a device of one's own design, a vacuum capsule, which was subject to certain technological constraints at the time of its creation. The most important element of this device was a vacuum collar, which was installed around the pelvis of the tested person, tightly, not permeable to air, but at the same time enabling the capsule to obtain the desired negative pressure. The negative pressure generated around the lower half of the body of the desired level allowed to simulate the orthostatic test, creating the conditions for transport of blood from the head to the lower parts of the body. A lot of papers prepared in the team, among others by dr. Dębiński, dr. Kuzak, dr. Żebrowski, were presented at national and international congresses (e.g. Reno, USA, 1996), and were very well received. As a result, MIAM was appointed to organize the World Gravitational Congress in Poland, which was held in 1997 with a very good result. 


\section{ORGANIZATIONAL ACTIVITY}

In 1984 I passed a specialization exam in the first degree of internal diseases at the Central Hospital for Veterans at Military Medical Academy (MMA). Specializations in internal diseases and previously passed 1st and 2nd degree exams in specializations in aviation medicine made it much easier to work in contact with the aviation personnel and allowed for undertaking a number of scientific works where technical knowledge was necessary. At the end of the 1990s I passed a specialization exam in transport medicine. In 1992, I became the Deputy Commander of MIAM for Medicine and I held this position until 1996.

In 1997, I was sent to work outside MIAM at the position of the Director of the Defense Department of the Ministry of Health, and then I was transferred to the Inspectorate of Health of the Ministry of National Defense to the position of Chief Sanitary Inspector - Deputy Head of Health Care of the Polish Army. At the end of the 1990s, I took up a job at the Main Military Aviation and Medical Commission, where I dealt with issues related to the causes of the inability of aviation personnel to serve in the air for health reasons. In the years 2001-2002, as a specialist in transport medicine, I organized courses for physicians who acquired the right to examine drivers and candidates for drivers, as well as for examination in order to obtain the right to possess weapons. I was then of- fered a position of the Head of the Aeronautical and Occupational Medicine Certification Center (AOMCC), which I took in 2002. It was a big organizational challenge, because it was necessary to organize the work of the center almost from scratch and create the scope of aviation and medical research. As the manager of a large area, I have set up a multi-specialist team to develop the principles of examinations of the aviation personnel, and to extend the methods of research to include modern research techniques. The result of many years of research was the Aviation and Medical Research Instruction prepared by the above mentioned team, submitted for evaluation to the Inspectorate of Military Health Care and the Ministry of National Defense.

In 2012, together with my colleagues from AOMCC, I took part in the development of the methodology of testing candidates for the Aviation High School, which is a preliminary selection of suitability for subsequent candidacy for the Air Force Officer School.

\section{DISTINCTION}

On the occasion of the 90th anniversary of WIML I was awarded in 2018 by the Director of WIML for my 50 years of work at MIAM.

\section{AUTHORS' DECLARATION:}

Study Design: Bronisław Turski; Data Collection: Bronisław Turski; Manuscript Preparation: Bronisław Turski. The Author declares that there is no conflict of interest. 